\title{
MATHEMATICAL, PHILOSOPHICAL AND SEMANTIC CONSIDERATIONS ON INFINITY (I): GENERAL CONCEPTS
}

\author{
J.L. Usó-Doménech, J.A. Nescolarde-Selva and M. Belmonte-Requena. \\ Department of Applied Mathematics. University of Alicante. Alicante. Spain.
}

\begin{abstract}
In the Reality we know, we cannot say if something is infinite whether we are doing Physics, Biology, Sociology or Economics. This means we have to be careful using this concept. Infinite structures do not exist in the physical world as far as we know. So what do mathematicians mean when they assert the existence of $\omega$ (the mathematical symbol for the set of all integers)? There is no universally accepted philosophy of mathematics but the most common belief is that mathematics touches on another worldly absolute truth. Many mathematicians believe that mathematics involves a special perception of an idealized world of absolute truth. This comes in part from the recognition that our knowledge of the physical world is imperfect and falls short of what we can apprehend with mathematical thinking. The objective of this paper is to present an epistemological rather than an historical vision of the mathematical concept of infinity that examines the dialectic between the actual and potential infinity.
\end{abstract}

Keywords: Actual infinity, Coincidentia oppositorum, Limit, Paradoxes, Succession, Potential infinity, Transfinite.

\section{INTRODUCTION}

Infinity is not an easy concept. For (Manfreda Kolar, V.\& Hodnik Čadež, T., 2012) a number of difficulties that people cope with when dealing with infinity include its abstract nature, the well-known paradoxes, and understanding infinity as an ongoing process which never ends, or as a set of an infinite number of elements. Infinity can be understood in several often seemingly incompatible ways, and involve value judgments or assumptions that are neither explicit nor desired. In what follows we distinguish several aspects, some potential, others actual and some teleological. Historically there were theological and mystical interpretations to the infinite (Restivo, 1963) ${ }^{1}$. Another

\footnotetext{
${ }^{1}$ For Jewish mysticism, the Infinity, Ein-Sof, the infinite God, has no static definable form. Instead, the Kabbalists conceive God, the world and humanity as evolving together through, and thus embodying, a number of distinct stages and aspects, with later stages opposing but at the same time encompassing earlier ones. The Kabbalist's God is both perfectly simple and infinitely complex, nothing and everything, hidden and revealed, reality and illusion, creator of man and created by man. As Ein-Sof evolves it is progressively revealed as "nothing whatsoever" (Ayin), the totality of being, the Infinite Will (Ratzon).Some Jewish writing (Kaplan 1990; Saks 1990, 2002; Schochet, 1979) refers to different levels of infinite spiritual worlds, an idea similar to Georg Cantor's idea that there is an infinite sequence of (mathematical) infinities: $\boldsymbol{N}_{0}<\boldsymbol{N}_{1}<\boldsymbol{\aleph}_{2}<\ldots$. Cantor's demonstration that the infinite cube contains exactly the same number of points as the one-inch cube helps to understand "the paradox of God's simultaneous transcendence and immanence."
} 
interpretation attributes infinity to Reality. Words implying all or any may be used synonymously with the infinite: every man, every triangle... However, there is a difference between these terms. The first refers to all men (who have existed and will exist) and is finite while the second, the number of triangles is not finite. Another aspect of the idea that we are trying to refine and specify is the concept very small. We consider that the very small is related to the very large, for obvious reasons. If big arises through a process of accumulation of one more iteratively (stacking), reverse the process countless times and the result will be small by shrinking.

In economic terms this feature could be equated with the property required by the economists of the school of Lausanne, thin divisibility. On the negative side we would have zero, not a negative infinite but an interpretation of a trend and the reverse of the growth path. Cognitive scientist George Lakoff (Lakoff \& Nuñez, 2000) considers the concept of infinity in mathematics and the sciences as a metaphor. This perspective is based on the basic metaphor of infinity (BMI), defined as the ever-increasing sequence $<1,2,3, \ldots>$. Perspective artwork utilizes the concept of imaginary vanishing points, or points at infinity, located at an infinite distance from the observer. This allows artists to create paintings that realistically render space, distances, and forms (Kline, 1985).

We should distinguish two aspects. One is the negative infinite, that represents the lack of something and this absence is glaringly and increasingly demanding and painful. Another aspect would be zero, the total absence of all being (understood in its broadest sense, as a way to exist) or any quality. These "concepts" are often misunderstood, as the total lack of goodness can be equated with total depravity, but they are different concepts, not having one does not mean having otherwise and not to be beautiful does not mean to be ugly.

The idea of the infinite seems difficult to grasp, because, at first sight, it is beyond all human experience. The human mind is acccustomed to dealing with finite things, reflected in finite ideas. Everything has a beginning and an end. This is a familiar thought. But what is familiar is not necessarily true. The history of mathematical thought has some highly instructive lessons on this score. For a long time, mathematicians, at least in Europe, sought to banish the concept of infinity. Their reasons for so doing are obvious enough. Apart from the evident difficulty in conceptualizing infinity, in purely mathematical terms it involves a contradiction. Mathematics deals with definite magnitudes. Infinity by its very nature cannot be counted or measured. This means that there is a real conflict between the two. For that reason, the great mathematicians of ancient Greece avoided infinity like the plague. Despite this, from the beginnings of philosophy, men speculated about infinity. Anaximander (610-547 B.C.) took it as the basis of his philosophy. In ancient Greek the term apeiron meant endless, unlimited, infinite, the unlimited or lacking definition, or without measure. Therefore, one could interpret the apeiron as indicating ethical chaos. Euclid avoided using the word infinite in its elements, saying "there is an infinite amount of... ". He preferred to use the phrase "an amount greater than any given". The Socratics associated the idea of infinite to something bad and evil. The infinite was not only huge, enormously large and indefinite, but was associated with the negative idea of disorder of chaos, the imperfect. Zeno's paradoxes (Huggett, 2010 ${ }^{\mathrm{a}, \mathrm{b}}$ ) do not prove that movement is an illusion, or that Achilles, in practice, will not overtake the tortoise, but they do reveal brilliantly the limitations of the kind of thinking now known as formal logic. The attempt to eliminate all contradiction from reality, as the Eleatics did, inevitably leads to this kind of insoluble paradox, or antinomy, as Kant later called it. In order to prove that a line could not consist of an infinite number of points, Zeno claimed 
that, if it were really so, then Achilles would never overtake the tortoise. There really is a logical problem here. As Alfred Hooper explains (Woods \& Grant, 2003, pp. 151): "This paradox still perplexes even those who know that it is possible to find the sum of an infinite series of numbers forming a geometrical progression whose common ratio is less than 1, and whose terms consequently become smaller and smaller and thus 'converge' on some limiting value."

In fact, Zeno had uncovered a contradiction in mathematical thought which would have to wait two thousand years for a solution. The contradiction relates to the use of the infinite. In the interpretation of classical mathematics the notion of limit (potential infinite) has been applied to the sum of the terms of a indefinitely decreasing geometric series giving a finite result for a sum of infinite terms (actual infinity). This is a contradiction. In our view, the reason for this contradiction lies in the assumption that we make as mathematicians, that in an indefinitely decreasing series, the term infinite reaches the (potential infinite) limit, or rather, that when we approach this Gimit, the value of $\mathrm{r}^{\mathrm{n}}$ becomes negligible. This is only justified if it were a practical measurement, since then, much as the measure is fine in the end, the measuring device is not able to record an infinitesimal increase in magnitude. However, the geometric space consists of infinite points, from which it follows that no amount, no matter how small, can be neglected, and Zeno's paradox lies precisely in this mathematical quality. So the assumption that we can approach infinity and it is not worth taking into consideration the value of $r^{\mathrm{n}}$, is opposite to the presumption that Zeno makes in his aporia, since, according to the Eleatics, the distance between Achilles and the Tortoise is always a geometrically straight segment, $d_{n}=d_{0} r^{n}$, which by definition contains infinite points (actual infinity). Thus, if we remain faithful to Zeno's thought, the final conclusion is that it is rationally impossible to reach infinity, this is only possible when $r^{n}=0$. As long as infinity not reached, we will, as Zeno says, have an infinite number of points (actual infinity) between Achilles' and the Tortoise's location. Then if actual infinity seems to be a property of rational thought, the notion of a limit (potential infinite) appears to be a mathematical concession to the world of the senses, a concession that would seriously upset Zeno, whobelieved that rationality prevails over the senses.

From Pythagoras right up to the discovery of the differential and integral calculus in the 17th century, mathematicians went to great lengths to avoid the use of the concept of infinity. Only the great genius Archimedes approached the subject, but still avoided it by using a roundabout method. The early atomists, starting with Leukippus, who may have been a pupil of Zeno, stated that the atoms "indivisible and infinite in number, move about ceaselessly in empty space, of infinite extent." The greatest of Greek mathematicians, Archimedes (287-212 B.C.) made effective use of indivisibles in geometry, but considered the idea of infinitely large and small as being without logical foundation. Likewise, Aristotle argued that, since a body must have form, it must be bounded, and therefore cannot be infinite. While accepting that there were two kinds of "potential" infinities_successive addition in arithmetic (infinitely large), and successive subdivision in geometry (infinitely small)- he nevertheless polemicised against geometers who held that a line segment is composed of infinitely many fixed infinitesimals, or indivisibles [see section 3]. This denial of the infinite constituted a real barrier to the development of classical Greek mathematics. By contrast, the Indian mathematicians had no such scruples and made great advances, which, via the Arabs later entered Europe.

For Dionysios the Areopagite there is no intellectual concept that can cover all features of God's infinity (actual infinite). Thus, he created apophatic theology in which all 


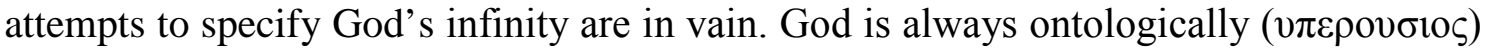
and epistemologically beyond the way humans think about Him.

The attempt to banish contradiction from thought, in accordance with the rigid schemas of formal logic held back the development of mathematics. But the adventurous spirit of the Renaissance opened men's minds to new possibilities which were, in truth, infinite. In his book The New Science (1638), Galileo pointed out that every integer (whole number) has only one perfect square, and every perfect square is the square of only one positive integer. Thus, in a sense, there are just as many perfect squares as there are positive integers. This immediately leads us into a logical contradiction. It contradicts the axiom that the whole is greater than any of its parts, inasmuch as not all the positive integers are perfect squares, and all the perfect squares form part of all the positive integers.

When mathematicians, speaking about infinite sets, use the expression, "all but a finite number of members," they are saying that the infinite "all" is still the same size "all" even if we take away some finite set. The removal of billions of members from an infinite set in no way diminishes its infinity. The least that can be said is that the infinity of the mathematicians supplies us with a useful metaphor: many of the assertions we make about God and about a mathematical infinity are similar. Modern physics accepts that the number of instants between two seconds is infinite, just as the number of instants in a span of time with neither beginning nor end. The universe itself consists of an infinite chain of cause and effect, ceaselessly changing, moving and developing. This has nothing in common with the crude and one-sided notion of infinity contained in the infinite series of numbers in simple arithmetic, in which "infinity" always "starts" with the number one.

This is only one of the numerous paradoxes which have plagued mathematics ever since the Renaissance when men began to subject thêir thoughts and assumptions to a critical analysis. As a result of this, slowly, and in the teeth of stubborn resistance from conservative minds, one by one the supposedly unassailable axioms and "eternal truths" of mathematics have been overthrown. It seems likely that only in the realm of pure mathematics can the idea of infinity be entertained. In the context of actual, manifest, realizable quantities things seem much more like the situation in a computer where all phenomena have definite resolution and size. One can never create an infinitely large file because that would require an infinite amount of time and infinite computational resources such as memory. Even with the domain of pure mathematics, infinities can only exist because they are symbolically represented and never actually represented. No one has ever written out an infinite number of integers thereby actually representing the set of integers. It is only ever referred to but never fully represented. If one required sets to be fully represented then mathematics could not operate on actual infinite sets; it could only operate on potentially infinite sets which always have finite representations (e.g., $\{1,2,3, \ldots\})$ but which are unlimited in their length. Such sets are arbitrarily large but always have a definite finite size. Modern mathematics is totally dependent upon the assumed existence of actual infinities but the existence of these can neither be proven nor disproven by mathematics. This leaves modern mathematicians in the position of defending their belief in the existence of actual infinities and discrediting any opposing ideas, but beside all of this - do actual infinites exist? All the evidence seems to suggest that they only exist within the context of modern mathematics, which would seem to suggest that modern mathematics should return to its axioms and see where the problem arises. 
We arrive at the point where the entire edifice has been shown to be unsound and in need of a thoroughgoing reconstruction on more solid, yet more flexible foundations, which are already in the process of being laid, and which will inevitably imply belief.

\section{PHILOSOPHICAL CONSIDERATIONS}

The concept of infinity from a philosophical perspective has been widely discussed as it leads to contradictions and paradoxes, from Euclid (the whole is not greater than the parts), Zeno's paradox (how does one halve the infinite in finite time?) $)^{2}$ or Russell (the set of sets that do not belong to itself) or the Hilbert Hotel ${ }^{3}$. In the seventeenth century, in what might be called the modern world, the idea was introduced of considering that the finite and the infinite are governed by different laws and precepts. John Wallis, in the seventeenth century introduced the symbol of infinity. Wallace may have been inspired by the Roman M which evolved from the earlier Etruscan symbol for 1000 resembling the lemniscate, as never ending lines. "Coincidentally" this symbol is used in the Tarot, designating perpetual motion. This letter is associated with the first letter of the Hebrew alphabet $\aleph$, used later by Cantor, who also related the idea of the infinite with God. Although attempts have been made to separate, or link, the infinite with certain ethical ideas, this has not been achieved completely and reappears in a cyclical manner. Examples are the considerations of S. Augustine and St. Tomas, for whom the infinite was a challenge to the One that could be considered infinite, that is, God.

The inclusion of the Potential infinity for the finite is aprimordial characteristic of the concept as it emphasizes that continua are indefinitely divisible.

\footnotetext{
${ }^{2}$ Zeno's paradoxes are a set of philosophical problems generally thought to have been devised by Greek philosopher Zeno of Elea (ca. 490-430 BC) to support Parmenides's doctrine that contrary to the evidence of one's senses, the belief in plurality and change is mistaken, and in particular that motion is nothing but an illusion. It is usually assumed, based on Plato's Parmenides (128a-d), that Zeno took on the project of creating these paradoxes because other philosophers had created paradoxes using Parmenides's ideas. Thus Plato has Zeno say the purpose of the paradoxes "is to show that their hypothesis that existences are many, if properly followed up, leads to still more absurd results than the hypothesis that they are one." (Parmenides 128d). Plato has Socrates claim that Zeno and Parmenides were essentially argaing exactly the same point (Parmenides 128a-b). Some of Zeno's nine surviving paradoxes (preserved in Aristotle's Physics and Simplicius's commentary thereon) are essentially equivalent to one another. Aristotle offered a refutation of some of them. Three of the strongest and most famous - that of Achilles and the tortoise, the Dichotomy argument, and that of an arrow in flight-are presented in detail below. Zeno's arguments are perhaps the first examples of a method of proof called reductio ad absurdum also known as proof by contradiction. They are also credited as a source of the dialectic method used by Socrates.

${ }^{3}$ Consider a hypothetical hotel with a countably infinite number of rooms, all of which are occupied. One might be tempted to think that the hotel would not be able to accommodate any newly arriving guests, as would be the case with a finite number of rooms. It is also possible to accommodate a countably infinite number of new guests: just move the person occupying room 1 to room 2, the guest occupying room 2 to room 4 , and, in general, the guest occupying room $n$ to room $2 n$, and all the odd-numbered rooms (which are countably infinite) will be free for the new guests. It is possible to accommodate countably infinitely many coach loads of countably infinite passengers each, by several different methods. Most methods depend on the seats in the coaches being already numbered (alternatively, the hotel manager must have the axiom of countable choice at his or her disposal). In general any pairing function can be used to solve this problem. For each of these methods, consider a passenger's seat number on a coach to be $n$, and their coach number to be $C$, and the numbers $n$ and $C$ are then fed into the two arguments of the pairing function.
} 
Example 1: The division of a segment of a line in disjointed parts is endless and the Potential infinity of these parts is enclosed within the segment.

That infinite Spinoza (2007) called the "infinity of reason", a suitable word as it raises the dignity of the concept. Whereas merely potential infinity, the divergent sequences, devoid of limit, Spinoza referred to as "the infinite of imagination", and it also appears judicious if one serves the operating mind, to be able to access such an infinite, and serves as much for imaginative representations inside of "mental space" as for analytical reasoning. The problem of potential knowledge of finitude, of its relationship with infinity is important as a foundation and condition of understanding possibility and potentiality. These are attitudes that the knowing subject can assume before disappearing under the shadow of the topic that Hegel (1990) recognized as crucial in this dialectical struggle, namely between infinity actu (actual infinity) and the cognitive attitude appropriate to understand it. However, infinite divergences are unleashed from the knowledge of the finite, with the obvious goal of the search for truth that involves, in effect, something deeper and more radical such as the definition of the relationship between finitude and infinity and, therefore, two opposing fundamental ideas concerning infinity. This, in turn, is the true touchstone of this debate, beginning with Spinoza, and extending its validity to all thought of modern times.

Hegel denounces the background fallacy of the Jacobian yision (Jacobi, 1980) in the confusion between the empirical infinitum (imaginative) and infinite actu in Spinoza, clarifying on the one hand, the characteristics and the fundamental role of the first infinite and, on the other, the essence of substance, making it indivisible, truthful and understanding, i.e., also of the particular or finite. This is probably the biggest difference between infinitum actu, that Spinoza defines as the "absolute affirmation of the existence of some nature and on the contrary the finite is its partial denial" (Spinoza, 2007), and the infinite empirical finite, sum of the individual, namely, for each of the subdivisions of the substance that appear exclusively absolute in the eyes of the Imaginatio (Hegel compares with reflective attitude of modern Verstand). The sum of the infinite negations of substance (the ordo rerum et connectio), the result of abstraction and hypostatizing by Reflection, is opposed to infinity which, in turn, becomes something "partially" denied. This attitude is the result of imaginative rapprochement, the typical approach of reflective dualistic philosophy which puts one as what is not what the other is, each thing at the same time in and for itself given as another, accordingly a string of denials made positive by imagination. The infinite actu, however, resolves this opposition in itself. The eternal, Hegel argues, is the absolute identity of the two (finite and infinite) which exceeds the absolute contradiction of empirical infinity itself, annihilating the opposition (finite/infinite) and the abstractions produced by Imaginatio.

It is fundamentally here that two different versions of the transcendent face each other: the traditional, proposing infinity as radically regarding the finite in its essence is concerned with establishing instantable opposition, and a new, original, where finite and infinite are no longer opposed. In the infinite the infinite series of finite modes of the substance, or its conditions, is collected and annulled in its apparently objective consistency.

1) The first vision corresponds with a qualitative incommunicability between finite and infinite (which for Jacobi are the natural world and God (Jacobi, 1980)) in which the only bridge to exceed that call Schranken (bearing in mind the 
Kantian distinction between Grenz and Schranke) is constituted by faith or, rather, by the famous somersault (Kant, 2002).

2) The second perspective, on the other hand, could be defined as reconciliation, in which the finite is not required to consider a radically other, but through an itinerary that could define gnoseolgical and certainly epistemological (from Imaginatio to Amor Dei intellectualis understood as looking from a high sensitivity to looking from the point of view of substance), may be recognized as resolved within the everything-finite.

As for the criticism of the confusion that would supposedly follow from Jacobi's two infinities or better, between these two approaches on Spinozian infinity, Hegel points to Jacobi's intention to attribute to Spinozan thought the concept of an empirical infinity strictly related to the absolutism of the finite.

This debate about the doctrine of Spinoza is a casus belli that Hegel uses to resolve important issues of his time and definitely made progress confronting some of the famous personalities and traditional philosophical views of the time. With this objective and aware of the value and weight that comes with pronouncing on thesematters Hegel uses the celebrated and debated geometric-mathematical example that Spinoza used to clarify the idea of infinitum actu. This example, which Hegel interprets and attacks Jacobi, is called Letter on the Infinite, because it explicitly clarifies the Spinozian difference between infinitum actu and infinite empirical or imagination (Edition by Paulus (1802-1803). In this letter, sent to Meyer on 20 April 1663 to clarify the essence and nature of infinitum actu, Spinoza uses an example that Jacobi considered questionable asserting that here Spinoza himself would have been misled by their own imagination (Jacobi, 1996).

\section{THE MATHEMATICAL INFINITY}

The problem of two mathematical infinities, the potential and the actual has divided schools from the time of ancient Greece. Aristotle in his Physics, gives an idea of the state of the discussion at that time. Our renewed confrontation among mathematicians as well as philosophers is due to Cantor with his theory of transfinite numbers, and new algorithmic forms of old actual infinity. Included in the philosophical discussions are ethical considerations that associate the infinite with the bad or, at least, with the uncanny (a view that still stands). These were overcome by distinguishing between actual and potentialinfinity.

\subsection{Potential infinity}

Potential infinity is an idea used in calculus. Potential infinity is characterized by the idea of one more. It represents a cumulative process. The fundamental idea of "the infinite" is that there is always one more (less), one behind (above). It should be noted that this conception of infinite indicates a trend, a behavior that never ends. Infinite potential can be considered as a teleological concept. Note that this idea is not about certain ethical principles of perfection. This conception of infinity corresponds to a teleological interpretation of the infinite. Indeed, teleology studies final causes and Potential infinity reaches towards the end, knowing that it will never come, because there is always more, whether numbers, steps, intervals... Infinite potential is linked to the repetition of a process that never ends, leading to many problems and paradoxes, from Achilles and the tortoise to Zeno, on the impossibility of movement (how to give infinite steps in a limited time?). This conception of the Potential infinity is flirting with 
the idea of limit, or rather with the operation of passage to the limit. Note that both concepts exist only as a tendency, in potential, since the two are inaccessible, which means that the terms infinite and unlimited should not be regarded as synonymous.

Potential infinity refers to a procedure that gets closer and closer to, but never quite reaches, an infinite end. For instance, the sequence of numbers $1,2,3,4, \ldots$ gets higher and higher, but it has no end; it never gets to infinity. Infinity is just an indication of a direction -- it's "somewhere off in the distance." Chasing this kind of infinity is like chasing a rainbow or trying to sail to the edge of the world -- you may think you see it in the distance, but when you get to where you thought it was, you see it is still further away. Geometrically, imagine an infinitely long straight line; then "infinity" is off at the "end" of the line. Analogous procedures are given by limits in calculus, whether they use infinity or not. For example, $\lim _{x \rightarrow 0}(\sin x) / x=1$. This means that when we choose values of $\mathrm{x}$ that are closer and closer to zero, but never quite equal to zero, then (sin $\mathrm{x}) / \mathrm{x}$ gets closer and closer to one.

\subsection{The actual infinity}

If we emphasize the "totality" of the concept of infinite as a unit, as one, we will have the current version of infinite. This idea corresponds to "that which no greater can be thought". Actual infinity is considered as a whole, such as the set of natural numbers, all, not some, but all of them. This conception of infinite arises when considering it as a unit. That is, we have (in the sense of unity) a "thing" that is infinitely large or numerous, as the natural numbers or numbers that are multiples of 11 . We treat it as if it was an item that comes to pass over the limit. It appears when we have arrived, when we have the total. This idea creates difficulties because we do not have an infinite, but many, which is difficult to compare and ultimately for measurement. Having admitted the existence of actual infinity, as many mathematicians refused to such as Cauchy and Gauss, it is easy to show that we have several infinites, which means that some are different than others and, therefore, of different size. That is, we will have some more infinite than others. Intuitively, if we consider the multiples of 11 and natural numbers, both are infinite, but it "appears" that the former is 11 times smaller than the second, but both are infinitely large. In logical terms we could say that the second is contained in the first and, given the postulate of Euclid, which states that the whole is greater than the parts, both infinites should be different, but they are not, they are the same size.

Cardinal is defined as the size of a set, and the cardinal of both sets is the same, as Cantor demonstrated. Cantor's argument is simple. Just make sure that you can establish a bijective correspondence ${ }^{4}$ between the set of natural numbers and the set of multiples of 11 , so that these sets are equipollent (have the same cardinal number). It is just as we can number the points of a semicircle or the points of a straight line. This is the definition that St. Anselm used with the idea of God.

Could one establish a bijective correspondence between real and rational numbers? It is therefore evident that the set of real numbers is not countable, while the set of rational is countable, so that the cardinals of both sets are different, that is, they are not equipollent. However, when we talk about natural numbers or points on a line, or a plane, or rational numbers or a Cantor set we are considering that each set is infinite, although their "sizes" are different (Tirosh, 1999; Tiroshand Tsamir, 1996). We need a definition for the size of these sets, the appeal to their number does not work, since, as

${ }^{4} f: \mathbb{N} \rightarrow \mathbb{N} ; \quad f(n)=11 \cdot n$ 
noted, the same "numero" (name) is used to describe different sizes and therefore different sets. To avoid these difficulties we define the infinite saying that a set is infinite if we can establish a correspondence between it and some of it. This definition is consistent with the existence of different infinites. To demonstrate this we simply apply Cantor's rule of the diagonal. Similarly it must not be concluded that when a set is contained in another set, the former is a lesser infinite. Consider the set of natural numbers and the even numbers. At first it may seem that there are twice as natural numbers of pairs. However, both sets have the same number of elements. This concept is clarified if size is understood as its cardinal set, so that other content set can have the same size as the first. In this sense the assumption that the whole is greater than the parts is broken. In "Euclidean" terms one could expand the postulate saying that the whole is greater than or equal to the parts.

\subsection{Cantorian axiomatic system}

It seems natural to ask if any two sets have or have not the same number of elements. For the case of finite sets, simply count the items in each set. But in the case of infinite sets, the answer depends on what is understood by sets with the same number of elements, i.e., equipotent.

Definition 1 (Postulate of cardinal number): For any set $X$ there exists an application $k$ with domain $X$ and such that if $Y$ is another set to verify that $k(X)=k(Y) \Leftrightarrow X \approx Y$.

For each set $\mathrm{X}$, the set image $\mathrm{k}(\mathrm{X})$ by the Tarskifunction $\mathrm{k}$ is usually represented by

Card (X), or $\bar{X}$ according to the notation created by Cantor and the two bars indicate the cardinal of $X$ obtained by two successive abstractions: the nature of the elements of $\mathrm{X}$ and the order in which these sub-elements are in $\mathrm{X}$. The postulate of Tarski, valid for sets is not essential in the sense that the cardinal can be elaborated later to the ordinal to defining the cardinal number with reference to the ordinal number. The interest in the suppression of this postulate comes mainly from aesthetics, and, secondarily, in that philosophers remain attached to the Cantorian work on order and not on his mathematical work (Cavailles, 1962).

Definition 2: Card $(X)$ is a cardinal number iff there is a set $X$ such that $k(X)=\operatorname{Card}(X)$

\section{Definition 3:}

1) $\operatorname{Card}(X) \leq \operatorname{Card}(Y) \Leftrightarrow X \leq Y$

2) $\operatorname{Card}(X)<\operatorname{Card}(Y) \Leftrightarrow X<Y$

Cantor's Theorem: $\overline{\bar{X}} \leq \mathrm{P}(\mathrm{\overline {X }})$ where $\mathrm{P}(\mathrm{\overline {X }})$ is the power set of $X$

Definition 4: $\operatorname{Card}(X \cup Y)=\operatorname{Card}(x)+\operatorname{Card}(Y)$ whenever $X \cap Y \neq \varnothing$.

Denoting two cardinal numbers by a and b, then the above definition would have to be specified in the direction. "There are disjoint sets $X, Y$ such that $a=\operatorname{Card}(X, b=$ $\operatorname{Card}(Y))$ and cardinal $X \cup Y$ would sum $a+b "$. This cardinal sum would be 
independent of the sets $\mathrm{X}$ and $\mathrm{Y}$, provided that they take with them equipotent sets. For this independence the cardinal sum would be unique.

Definition 5: $\operatorname{Card}(X \times Y)=\operatorname{Card}(x) \cdot \operatorname{Card}(Y)$

The first four postulates of Peano (1979) intend to found an order of succession, natural for Integers. However Russell (1903) objected that Peano defined only one type of order in any sequence that was "well-ordered" without repeating any terms, and indefinite. Here is an example of a sequence belonging to these types of order: $\sqrt{\frac{1}{2^{2}}}, \sqrt{\frac{2}{3^{2}}}, \sqrt{\frac{3}{4^{2}}}, \ldots$. It properly belongs to the order genre defined as a Peano series of natural integers, but it is also common to countless other sequences. As well, these postulates are not just quantitative: the order is a quality relationship. The amount that an integer expressed was not constructed by Peano, rather he was concerned with the postulates that define the quantitative relation of order $>$. Just then the integer is formed with its two poles, the qualitative or ordinal and quantitative or cardinal. Then it appears that the natural order was none other than the increasing quantities. The natural sequence forms an arithmetic progression, where each term is generated by the addition of a unit to its predecessor. As specification of categorical bipolarity quality-quantity, we can conjecture that ordering and cardinality are also bipolar, which, peculiarly, are involved mutually in comprehension. This is in fact demonstrating the reconstruction of Peano: the main postulate the adding states that if $m+n=p$, then the addition to $m$ of the successor of $n$ will result in the successor of $p$, therefore the natural ordering includes comprehension of cardinality and, reciprocally, such ordering comes at the end of the relation>and is essentially quantitative, cardinal. For this reason, in the presentation of the Peano, cardinality is separated from ordination that is tied up to a kind of general, abstract formulation, not characteristic of natural sequence of integers. Otherwise, we are aware that by implication the reciprocal, and the inseparability, is maintained in transfinite numbers: the various constituted theorems of cardinality are required to demonstrate completely the postulate of choice and even the well-ordering theorem and, conversely, two infinite sets of identical order types have the same cardinality. Nevertheless, the sequence of natural integers is used to represent the order of all other sequences of the same type, to number their terms, even if the predecessor exceeds the successor, and even if these terms are unconnected material objects, the quantitative comparison of which makes no sense. The usual discourse asserts that in this last the ordering operator is the integer itself; but this is falling into tautology.

What is the most important pole, the ordinal or cardinal? Since the integers integrate real numbers which are not ordering operators, that alone justifies the choice in favor of the cardinal. While the specialized field of stream mathematics, integer or not, the real number is the most refined and therein lies much of its essence. It is also saying that if physical greatness are qualities that are quantified, then the pure numbers are quantities that are qualified, especially discrete integer amounts, discontinuous and real numbers that are continuous quantities.

Cantor's ideas that some infinites were more infinite than others, in addition to creating this scandal this view led to the formalization and extension of certain concepts such as cardinality and ordinal. We will not delve into these concepts as they are well known. It is just the point out that the cardinal indicates the unordered set size. That is, ignoring the order. The set of natural numbers Cantor appointed with the name of $\aleph_{0}$. The ordinal 
we report for the size of the set when elements of it are well sorted. That is, all the nonempty part has a minimal element. The first transfinite ordinal Cantor appointed it by $\omega$. It is curious that the Jesuit paleontologist Teilhard de Chardin, also speaks in his works of the $\omega$ point, though in a different sense: theological. Once we have the first transfinite we obtain the following with nothing more than to add to it , so we get $\omega+, 1 \omega+2, \ldots, \omega$ $+\omega=2 \omega, \ldots, \ldots, \omega . \omega . \omega . \omega . .$. Cantor asks and did not solve the problem of the continuum between $\aleph_{0}$ the cardinal of integers and $\aleph_{0}$ the cardinal of real numbers. Should there be any intermediate cardinal? Gödel and Cohen showed that the continuum theory can be taken as true or not without affecting set theory. The actual infinity is not only paradoxical, but it is consistent, which will force us to make a review of the development, use and review of the concept of infinity.

Now the door was open to the creation, and the naming, of a whole gamut of infinities, in fact, an infinity of infinities (Aczel. 2000). A new world of transfinite numbers was being created. In according to Graham and Kantor (2009) should we say, "the oreation and the naming" of transfinite numbers or "the naming and the creation" of them? Which comes first?

The axiomatic system chosen corresponds to the Neumann-Bernays-Gödel system modified by Morse. For the authors, this is the most important operating system for current Mathematics. For mathematical considerations we consulted the following authors: Bourbaki (2008), Cantor (1955), Denjoy (1949-1954), Fraenkel (1939, 1949, 1953), Hausdorff (2005), Kamke (1950), Krivine (1969), Rubin (1967) and Supper (1960). Theorems, represented by T, do not include proofs, which are well known to mathematicians.

To Cantor (1955) is due the following definition.The set $A$ is equipotent to set $B$ and is denoted by $A \approx B$ if there exists a function $F: A \rightarrow B$ injective and surjective. It has been demonstrated that the relationship between sets defined $A \approx B$ is an equivalence relation. On the basis of the natural number $N=\{1,2,3, \ldots\}$ it is stated that if a set $A$ is equipotent to the set of natural numbers $N$ is called countable and is said to have the cardinal $\boldsymbol{N}_{0}$. Also, if a set is finite is also said to be countable and equipotent to a subset of $\mathrm{N}$. If a set is infinite and is not equipotent to $\mathrm{N}$ it is said to be uncountable. The relation of equipotency between sets verifies the properties of a relation of equivalence. It seems then natural to consider the class of all equipotent sets with one given as a new object. This is the process that allows the creation of new objects and therefore new sets and mathematical Structures. However, this consideration is confronted with certain difficulties. Thus, the equivalence relation must be defined in the "set of all sets", which is prohibited. This can be remedied by saying that is defined in its own class of all sets. Thus, the new object required to be in accordance with the equivalence relation itself, which is a set, but the establishment of this fact is only feasible accepting some kind of new postulate. From a pragmatic approach that is interesting, in the acceptance of this new postulate, each set is associated with a new set, the natural number, so verifying that it corresponds to equipotent sets with the same cardinal number. That is, what is important to know is how to manage the new object, and the rules that apply to it, rather than the search for the essence of this new object. It was Tarski that made it possible for this approach, valid for an intuitive theory in relation to axiomatic sets, to be accepted as a new postulate (Suppes, 1960).

Definition 6: A set is said to be infinite when is not finite. A cardinal number is said to be infinite when it is not finite. 
The natural numbers are a set $\mathrm{N}$, also represented by $\omega$.

Postulate of Infinity: There is an infinite set. The finite cardinals constitute a set N. $\exists X(\varnothing \in X \wedge \forall Y(Y \subset X \Rightarrow Y \cup\{Y\} \subset X))$

Dedekind's Definition: The necessary and sufficient condition that a set is infinite is that it is equipotent to any of its parts.

Finite sets have different properties from sets that are not finite. The definition given by Dedekind of an infinite set is based on equipotency of a set with its parts. And from this definition can be obtained the remaining properties.

T1: All subsets of $N$ are finite or equipotent to $N$.

T2: All infinite sets contain a subset that is equipotent to $N$.

\section{Definition 7:}

1)A set is said countable when it is equipotent with $N$.

2)A set $X$ is at its most countable when it is finite or countable.

3)A family of sets $\left(X_{i}\right)_{i \in L}$ is said to be countable, if their index set $L$ is countable.

T3: All subsets of $N$ are at their most countable.

T4: All infinite sets contain a countable subset.

T5: Any part of a countable set is at its most countable.

T6:

1) $N x N \approx N$

2) If $X$ and $Y$ are countable, so also is $X x Y$.

T7: If $\left(X_{i}\right)_{i \in L}$ is a countable family of countable sets, their union is also countable.

T8: $Q$ is countable.

T9: If $X$ is a countable set and $n$ is a natural number $n \geq 1$, then $X^{n}$ is numerable.

T10: If $X$ is a countable set and $n f$ is a surjective application of $X$ in $Y$, then $Y$ is at most numerable.

T11: If $\bar{X}_{\text {is }}$ infinite and $Y$ numerable, then $X \cup Y$ is equipotent with $X$ when ever $X \cap Y=\varnothing$.

The above theorems can be reformulated as propositions about natural numbers if we remember that the cardinal of natural numbers is designated by $\aleph_{0}$, as the first transfinite cardinal when $a=a+1$,or also, when there is an infinite set in the sense of the definition of Dedekind, such that its cardinal is $a$. With the convention that $\mathrm{n}$ designates the cardinality of a countable set, we have the following propositions in cardinal language:

T12: $\forall n, n \in N$, with $n<\aleph_{0}$ if $n$ is finite.

T13: $\forall a(\exists b, b \leq a)$

T14: $\aleph_{0} \cdot \aleph_{0}=\aleph_{0}^{2}=\aleph_{0}$

T15: 

1) $\aleph_{0}+\aleph_{0}=\aleph_{0}$
2) $\sum \aleph_{0}=\aleph_{0}$

T16: $\operatorname{Card}(Q)=\aleph_{0}$

T17: If $a \neq 0$ then $\aleph_{0}^{n}=\aleph_{0}$.

T18: $\aleph_{0}+a \leq a$

T19: $\aleph_{0} \leq 2^{\aleph_{0}}$

T20: The set $[0,1]$ is uncountable.

Definition 8: The sets that have the same power as $[0,1]$ are said to have the power of continuum and is denoted by $c$.

\section{THE HYPOTHESIS OF CONTINUUM}

Aristotle also analyzes the problem of the nature of a continuum ("continuum" being the Latin noun formed from the adjective "continuus", meaning (joinling, connected, uninterrupted or unbroken). Is a continuum, composed of indivisible elements or not? Most philosophers before Cantor, followed Aristotle in arguing that continuity was not composed of indivisible elements. In (Book V3 of the Physics) Aristotle defined various concepts including: Together, between, successor, contiguous and the concepts of continuous as a subdivision of the contiguous. "Things are called continuous when the touching limits of each become one and the same- and are contained in each other. Continuity is impossible if these extremities are two". In (Book VI.1 of the Physics) he argues that extremities of two points cannot be one, nor together. If the continuum consists of points, they should be in contact or continuous with each other. But if they are in contact, they will have different parts, while the continuum has different parts. Thus, the continuum is divisible only by parts that are infinitely divisible (231 b15).

Using the Cantorian hypothesis of continuum:

$$
\begin{aligned}
& \exists a\left(N_{0}<a<2^{\circ}\right) \\
& \exists a\left(N_{0}<g<c\right) .
\end{aligned}
$$

we obtain the following theorems:

T21: If $a<b$ then $[0,1] \approx[a, b]$.

T22: The intervals $[0,1[] 0,1],] 0,1[$ have the same power as $[0,1]$

T23: The set of real numbers $R$ has the power of a continuum.

T24: The power $P(N)$ of is $c$, e.g. $c=2^{\aleph_{0}}$

T25: The union of an infinite succession of disjoint sets of the power of continuum has the power of a continuum.

T26: $c \cdot c=c^{2}=c$.

T27: $c^{n}=c, \forall n \in N$.

According to Fischbein et al. (1979) the world of $\aleph_{0}, \aleph_{1}, \aleph_{2}, \ldots$ is composed of actual infinity and represents a potential, not an actual form of infinity.

Transfinite induction, like regular induction, is used to show a property $P(n)$ and holds for all numbers $n$. The essential difference is that regular induction is restricted to the 
natural numbers, which are precisely the finite ordinal numbers. The normal inductive step of deriving $P(n+1)$ from $P(n)$ can fail due to the limits of ordinals.

Let A be a well-ordered set and let $P(x)$ be a proposition with domain A. A proof by transfinite induction uses the following steps (Gleason 1991, Hajnal 1999):

1. Demonstrate $P(0)$ is true.

2. Assume $P(b)$ is true for $\forall a<b$.

3. Prove $P(a)$, using the assumption in (2).

4. Then $P(a)$ is true for $\forall a \in A$.

\section{ACTUAL INFINITY AND COINCIDENTIA OPPOSITORUM: CUSANUS AND CANTOR}

Greek philosophy, before the Christian theologian Gregor of Nyssa, was oriented towards finiteness. St. Augustine in his Civitate Dei conceived God as being infinite, this is a break with Aristotle's metaphysical concept of a finite God. This new concept of an infinite God raised the issue of how think about this infinity, which was inconceivable in classical Greek metaphysics. The theology of Dionysios the Areopagite and Gregor's theology stresses the infinity of God. However, Dionysios argues that this infinite cannot be captured in language. This apophatic answer raises the intellectual challenge to think and relate to the infinity of God, yet it is so spiritually powerful that it conveys the risk of eroding human andiivine rationality. God and the apophatic theology ${ }^{5}$ as a form of pious adoration of this infinity might have resulted in an intellectual dead end.

Cusanus, established a way for the intellectuâl understanding of the infinity of God through the symbolic use of mathematics. Cusanus was deeply influenced by Areopagite's apophatic approach and the work of Dionysius, who is the most cited author in the writings of Cusanus. He agrees with the Areopagite that God is infinite (Cusanus, 1986) and introduces a new thought about this infinity. Cusanus himself calls this thought as "never heard before" (prius inaudita). He qualifies the infinity of God as the coincidentia oppositorum ${ }^{6}$, the falling together of contradictions or opposites.

\footnotetext{
${ }^{5}$ Apophatic theology, also known as negative theology, is a theology that attempts to describe God by negation, to speak of God only in absolutely certain terms and to avoid what may not be said. In Orthodox Christianity, apophatic theology is based on the assumption that God's essence is unknowable or ineffable and on the recognition of the inadequacy of human language to describe God.

${ }^{6}$ The doctrine of coincidentia oppositorum, the interpenetration, interdependence and unification of opposites, $v(P \wedge \neg P)=1$ has long been one of the defining characteristics of mystical (as opposed to philosophical) thought. Mystics of various persuasions have generally held that such paradoxes are the best means of expressing within language, truths about a whole that is sundered by the very operation of language itself. Any effort, it is said, to analyze these paradoxes and provide them with logical sense is doomed from the start because logic itself rests upon assumptions, such as the principles PNC (Principle of no contradiction) and PEM (Principle of excluded middle), that are violated by the mystical ideas. The coincidentia oppositorum is a common trope in many religious traditions, particularly those with a mystical or initiatory aspect (Usó-Doménech, Nescolarde-Selva, Pérez-Gonzaga and Sabán, 2015).
} 
Cantor revived the tradition of Cusanus. Although a more comprehensive test would also mention Bernardus Bolzano (1781-1848), author of a treatise on paradoxes of infinity, and that it was really Georg Cantor who, in the late nineteenth century, realized such plurality, laying the foundations of set theory and transfinite numbers.

\section{REFLECTIONS}

Infinity was created by an axiom of existence and by a word that is ambiguous. If "there is infinity", finite ordinals form a set. Cantor has drawn a line where there is a man (infinity), and where there is no man (infinite). He does not stop there: by placing each power of infinity, in all parts of a set, he creates the infinite series of the transfinite. Without that we can reach a final transfinite, the cardinality of the set of all sets: this is a paradoxical set, because according to Russell, it would be a part to the set of the sets that do not belong, which either it belong to (and thus do not belong) or does not belong (and therefore belongs).

Transfinite sets have the property of reflexivity: they are coordinatable with parts (the integers are coordinatable with squares). How could we know the world if we were not coordinatable with it? Because we are so we can create: we can put borders (say no) and transgress. And that is because we have been made with border fences, the "nothing". Because we are a one generated by a zero, we are open to infinity.

The introduction of the notions of infinity in natural or real numbers, or a reference to the continuous, should be general epistemological criteria, and theoretical and functional requirements. The existence of transfinite wholes does not depend on rules to ensure formal consistency, or symbolizations, allowing construction in an algorithmic process. Is it also not the case that people can deny transfinite wholes in principle, or conversely use them without clear theoretical criteria? The first attitude (simple denial) impedes progress in mathematical practice; the second, which is the way that addresses logicism, leads to a path full of uncertainty.

Again the discussion comes to philosophy. Platonism is present in the logicism project in a very profound way. The same quantification applies to various wholes without providing appropriate criteria for the validity of its introduction.

\section{Acknowledgments}

The authors like to thank Dr. Hugh Gash for his important collaboration in this work, especially English corrections and scientific recommendations.

\section{REFERENCES}

Aczel, D, A. 2000. The mystery of the Aleph. Mathematics, the Kabbalah and the Search for Infinity. Pocket Books. A division of Simon\&Schuster Inc. New York.

Bandmann, H. 1992. Die Unendlichkeit des Seins. Cantors transfinite Mengenlehre und ihre metaphysischen Wurzeln. Frankfurt. (In German)

Bourbaki, N. 2008. Elément de Mathématique. Théorie des ensembles. Springer. (In French). 
Cusanus, N. 1986. Of Learned Ignorance, Germain Heron, tr. Yale University Press. New Haven.

Cavailles, J. 1962. Philosophie matyhématique. Hermann. Paris. (In French).

Denjoy, A. L'énumeration transfinite.1949-1954. Gauthier-Villars. Paris. (In French). Fischbein, E, Tirosh, D. and Hess, P. 1979. The intuition of Infinity. Educational Studies in Mathematics. 10, pp 3-40.

Fraenkel, A.1939. Natural Numbers as Cardinals. In Scripta Mathematica VI (2).

Fraenkel, A.1940. Natural Numbers as Ordinals. In Scripta Mathematica VII (1-4).

Fraenkel, A.1953. Abstract Set theory. Amsterdam: North Holland Publishing Co.

Gleason, A. M. 1991. Fundamentals of Abstract Analysis Natick, MA:A K Peters, p. 82,.

Graham, L and Kantor, J.M. 2009. Naming Infinity: A True Story of Religious Mysticism and Mathematical Creativity. Harvard University Press.

Hajnal, A.; Hamburger, P.; Bruce, J. W. (Eds.). 1999. Set Theory. Cambridge, England: Cambridge University Press, p. 66.

Hausdorff, F. 2005. Set theory. American Mathematical Society. AMS Chelsea Publishing.

Hegel, G.W.F. 1990. Glauben und Wissen en Gesammelte Werke, In Verbindung mit der Deutschen Forschungsgemeinschaft herausgegeben von der NordrheinWestphälischen Akademie derWissenschaften, Bd.2. Hamburg: Felix Meiner. (In German).

Huggett, N. 2010 . Zeno's Paradoxes: 3.1 The Dichotomy. Stanford Encyclopedia of Philosophy. Retrieved 2011-03-07.

Huggett, N. 2010 ${ }^{\mathrm{b}}$. Zeno's Paradoxes: 3.2 Achilles and the Tortoise. Stanford Encyclopedia of Philosophy. Retrieved 2011-03-07.

Jacobi, F.H. 1980. Über die Lehre des Spinoza, in Briefen an Herrn Moses Mendelssohn. Darmstadt: Wissenschaftliche Buchgesellschaft. Traducción española en Jacobi, F.H. 1996. Cartas a Mendelssohn. David Hume. Carta a Fichte. Barcelona, Círculo. (In Spanish).

Kamke, E. 1950. Theory of Sets. (F. Bagemihl, trans.). Dover. New York.

Kant, I. 2002. Crítica de la razón pura. Trad. de Manuel García Morente. Madrid, Tecnos. (In Spanish).

Kline, M. 1985. Mathematics for the nonmathematician. Courier Dover Publications. 
Manfreda Kolar, V. \& Hodnik Čadež, T. (2012). Analysis of factors influencing the understanding of the concept of infinity. Educational Studies in Mathematics. 80(3), pp 389-412.

Kaplan, A. 1990. Innerspace. Brooklyn, NY: Moznaim

Krivine, J.L. 1969. Théorie axiomatique des ensembles. PUF. Paris. (In French)

Lakoff, G. and Nuñez, R. 2000. Where Mathematics Comes From. Dover. New York.

Peano, G. 1979. Los principios de la aritmética: expuestos según un nuevo método. Julián Velarde Lombraña. ed. Traducido por Julián Velarde Lombraña (1 ${ }^{\text {a }}$ edición). Pentalfa Ediciones. (In Spanish).

Restivo, S. 1983. The social relations of Physics, Mysticism and Mathematics. D. Reidel Publishing Company. AA Dordrecht. Holland.

Rubin, J. 1967. Set theory for the mathematician. Holden Day. New York.

Russell, Bertrand. 1903. Principles of Mathematics, Cambridge: Cambridge University Press.

Saks, T. V. 1990. Applications of mathematical infinity in Jewish philosophy. In $\mathrm{H}$. Branover, A. Gotfryd, S. Lipskar (Eds.), Fusion: Absolute standards in a world of relativity, pp. 123-142. Jerusalem, Israel: Feldheim.

Saks, T. V. 2002. Different levels of infinity in Torah and mathematics. B'Or Ha'Torah, 13E, 113-120.

Schochet, J. I. 1979. Mystical concepts in Chassidism. Brooklyn, NY: Kehot Publication Society.

Spinoza, B. 2007. Etica demostrada según el orden geométrico. Madrid: Alianza. (In Spanish).

Spinoza, B. Opera. Edited by H. E. G. Paulus. 2 vols. (edition used by Hegel). Jena, DE. 1802-1803.

Suppes, P.1960. Axiomatic set theory. Van Nostrand.

Tirosh, D. 1999. Finite and infinite sets. International Journal of Mathematical Education in Science and Technology, 30, 341-349.

Tirosh, D. and Tsamir, P. 1996. The role of representations in students' intuitive thinking about infinity. Journal of Mathematical Education in Science and Technology, 27, 33-40. 
Usó-Doménech, J. L.; Nescolarde-Selva, J.; Pérez-Gonzaga, S; Sabán, M.J. 2015. Paraconsistent multivalued logic and coincidentia oppositorum: evaluation with complex numbers. American Journal of Systems and Software. 1 (3). pp. 1-12.

Woods, A. and Grant, T. 2003. Reason in Revolt: Dialectical Philosophy and Modern Science. Vol II. Algora Publishing.

\section{Author Biography}

José-Luis Usó-Doménech graduated in Sciences in 1968 and Doctor in Mathematics by the University of Valencia(Spain) in 1991. Since 1991, he has been working in the Department Mathematics, University of Castellon, Spain. He is currently retired. He made his doctoral thesis on a mathematical model of a terrestrial Mediterranean ecosystem. He has directed many doctoral thesis in Theory of Systems. He is author and co-author of several papers in journals, proceedings and books. He has obtained scholarships of research in many universities (University of Joensuu, Finland; University of Ben Gurion of Negev, Israel and the University of Georgia, USA). He has collaborated in numerous occasions with the Wessex Institue of Tecnology(UK) and fruit of this collaboration was the creation of the international congress ECOSUD.

Josué Antonio Nescolarde Selva graduated in Mathematics from the University of Havana (Cuba) in 1999. He won the award: Gold Title at the University of Havana, Cuba in 1999. He received the $\mathrm{PhD}$ degree in Mathematics from the University of Alicante(Spain) in 2010. Since 2002, he has beenworking in the Department of Applied Mathematics, University of Alicante, Spain. He is author and co-author of several papers in journals, and books. He is devoted to research on the Theory of Systems.

Mónica Belmonte Requena graduated from the University of Alicante in 2012. She received the MSc degree from the Oniversity of Alicante(Spain) in 2013. She is author and co-author of several papers in journals. 